\title{
Current concepts in the rehabilitation of pediatric traumatic brain injury
}

\author{
Linda E. Krach • Nanette Aldahondo • \\ Angela Sinner · Susan Quigley
}

Published online: 28 February 2013

(C) Springer Science + Business Media New York 2013

\begin{abstract}
Pediatric traumatic brain injury (TBI) is a common condition. Recent advances in this area have included the development of quality of care indicators for the rehabilitation of children with TBI, efforts to identify means to prognosticate regarding outcomes including results of biomarker and imaging assessments, innovations in rehabilitation techniques including the application of various technologies, and continuing delineation of longterm outcomes. Application of the quality of care indicators support that pediatric-specific rehabilitation units are more likely to meet the quality of care indicators than all age units or those that admit a small number of children with TBI. Some biomarkers are showing promise, particularly when considering peak concentration, but extracranial injury makes their use challenging. Long-term outcome studies report continuing impairment years after injury. Evidence is still limited and would benefit from the application of standard definitions and use of consistent outcome measures.
\end{abstract}

\section{E. Krach $(\bowtie)$}

Department of Physical Medicine and Rehabilitation,

University of Minnesota, 420 Delaware St. SE MMC 297,

Minneapolis, MN 55455, USA

e-mail: Krach002@umn.edu

N. Aldahondo $\cdot$ A. Sinner $\cdot$ S. Quigley

Gillette Children's Specialty Healthcare, 200 East

University Avenue, St Paul, MN 55101, USA

e-mail: naldahondo@gillettechildrens.com

\section{A. Sinner}

e-mail: asinner@gillettechildrens.com

S. Quigley

e-mail: squigley@gillettechildrens.com
Keywords Brain injury - Child · Rehabilitation · Outcome $\cdot$ Cognition disorders · Attention - Recovery of function - Standards - Data collection - Biologic markers · Computers: hand held · Executive function - Magnetic resonance imaging: methods $\cdot$ Prognosis

\section{Introduction}

Pediatric traumatic brain injury (TBI) is common condition. The CDC notes that in the US there are approximately 500,000 emergency department visits annually to evaluate children ages $0-14$ for TBI. The age groups with the greatest likelihood of sustaining a TBI include ages 0-4 and 15-19 in addition to adults over age 65 [1]. A recent report of the prevalence of TBI from a birth cohort of individuals between the ages of birth and 25 years in New Zealand indicated that the average incidence was from 1.10 to 2.36 per 100 per year with an overall prevalence of approximately $30 \%$ with $10 \%$ meeting criteria for moderate to severe injury [2]. There has been significant research interest and publication in this area. Areas of interest that will be reviewed in this article include quality indicators for pediatric TBI rehabilitation, innovations in imaging and biomarkers, application of technologies to this diagnostic group, and new information about long term outcomes.

\section{Quality indicators}

Rivera et al. [3] reported on the development of quality of care indicators for the rehabilitation of children with TBI. The goal of the project was to develop a measurement tool to assess compliance with processes of care for this 
population combining a systematic literature review to grade the relevant evidence and the use of the RAND/ UCLA Appropriateness Method and a Delphi technique with a national expert panel of nine geographically distributed pediatric physiatrists. Eight domains of management including general management; family-centered care; cognitive-communication, speech, language and swallowing impairments; gross and fine motor skill impairments; neuropsychological, social and behavioral impairments; school reentry; and community integration were identified.

This group then made use of these indicators to examine the variations in the structure and organization of care among a sample of rehabilitation programs identified using data from the National Association of Children's Hospitals and Related Institutions, Uniform Data System of Medical Rehabilitation and the Commission on Accreditation of Rehabilitation Facilities. Of the 74 inpatient units that treat children that were identified, 31 pediatric units and 28 all age units responded to a 12-question survey that was developed from the indicators noted above. Pediatric specialty units generally treated more than 20 children with TBI per year and had at least one child with TBI on the unit over $90 \%$ of the time and also had more pediatric specialty trained staff. Those centers with a higher number of children with TBI also had more resources. Overall, being a pediatric-focused inpatient unit and admitting a large volume of pediatric TBI patients were associated with the total number of quality indicators met [4].

An additional study was done including chart abstraction related to seven domains of acute rehabilitation care at nine institutions. One hundred and seventy-four patient records were studied. They found significant within and between institution variations in the percentage of individuals receiving recommended care for each of the domains. Institutions with pediatric specialty trained therapists had a higher adherence to motor, neuropsychological and community quality indicators. Those that only admitted children scored higher on cognitive, neuropsychological and school reentry domains, and CARF accreditation was associated with adherence in the school reentry domain [5•].

\section{Biomarkers}

Although different medical specialties make use of biomarkers to diagnose, direct treatment, and predict outcome, to date no single brain-specific biomarker has been established for use in routine clinical practice for TBI. In recent years, there has been significant research centered on finding clinically useful biomarkers for pediatric TBI.

Kövesdi et al. [14] and Papa et al. [6] outlined expectations regarding biomarkers. Biomarkers should be highly specific and sensitive for brain injury, appear rapidly in an accessible biological fluid and show a well-defined distribution in time, be proportional to the mechanical impact and extent of injury allowing stratification by injury severity and information about injury mechanism, reflect differences between age and sex groups, monitor progress of disease, response to treatment and assist in prognosis.

Neurospecific biomarkers that have been most frequently studied include: S100B, neuron-specific enolase (NSE), myelin basic protein (MBP) and glial fibrillary acid protein (GFAP).

S100b

$\mathrm{S} 100 \mathrm{~B}$ is the most extensively studied biomarker in TBI. Several studies have examined the usefulness of serum $\mathrm{S} 100 \mathrm{~B}$ in the management of mild TBI. Bouvier et al. [7] examined a group of 446 children $<16$ years of age with closed head trauma and varying severity as defined by the Masters classification. They reported significant linear increase $(p=0.013)$ in serum S100B concentrations according to injury severity. The S100B level was also found to be a highly significant indicator for correct stratification of patients into bad versus good 24-h clinical evolution groups $(p=0.0001)$. In addition, the S100B level correctly identified patients with trauma-relevant intracranial lesions with a sensitivity of $100 \%$ but with a specificity of $33 \%$. A proposed algorithm was created as a result of this study integrating $\mathrm{S} 100 \mathrm{~B}$ use in clinical decision-making. In another study of 146 children with mild TBI (mTBI) versus contusion, Geyer et al. [8] found no significant difference in mean S100B concentrations. Bechtel et al. [9] highlighted the limitations of S100B as a screening tool for intracranial injury in children who present with closed head trauma, particularly if they have multiple injuries due to multiple extracranial sources of S100.

Žurek and Fedora [10] found S100B levels were significantly higher at presentation and decreased significantly more slowly in patients with worse outcomes based on a Glasgow Outcome Score (GOS 4 or death). Berger et al. [11] examined initial and peak concentrations and demonstrated that peak concentration of S100B correlated more strongly with a 4-6 month GOS-Extended Pediatric score. They hypothesized that initial biomarker concentrations are representative of primary injury severity and accompanying cell death, whereas peak concentrations represent ongoing cell death including secondary injury. In this study, combining information about all of the biomarkers studied (S100B, NSE and MBP), they found negative and positive predictive values regarding outcome were 97 and $75 \%$, respectively. Due to previous findings demonstrating a lower specificity of S100B in younger children, Berger 
et al. analyzed their results in children $\leq 4$ and $>4$ years and found the correlation coefficients for initial and peak S100B were still significant but slightly lower in younger subjects.

In a 2010 review, Sandler et al. [12] note the differences in inflicted versus non-inflicted TBI with several studies showing worse outcomes in the inflicted TBI group and longer time-to-peak concentrations of biomarkers, including S100B. It remains important to highlight as Sandler et al. [12] did in a recent review that the specificity of S100B remains limited by the multiple extracranial sources of S100.

\section{Neuron-specific enolase (NSE)}

Geyer et al. [8] reported similar findings for NSE after mTBI as those seen with S100B.

In a prospective observational study of 63 pediatric patients, Žurek and Fedora [10] found NSE levels were significantly higher in those patients who died or had poor outcome at 6 months post-injury. Chiaretti et al. [13] studied CSF concentrations of NSE, nerve growth factor (NGF) and doublecourtin (DCX) in 32 children with TBI and found at $2 \mathrm{~h}$ post-injury the concentrations were dramatically increased in those with TBI as compared to controls by 42-, 22-, and 9-fold $(p<0.01)$. Up-regulation of NGF and DCX, relative to low NSE soon after injury seemed to predict better neurologic outcome in this group of children. In a Berger et al. [11] study, peak NSE concentrations correlated with outcome more strongly than initial concentrations. This is similar to findings with S100B. Additionally, the number of hours NSE remained in the abnormal range also correlated strongly with outcome at 0-3 months. When examining age group differences, the correlation between initial and peak NSE concentrations was much stronger in children $\leq 4$ years, but virtually no correlation was found with older children.

Sandler et al. [12] note in their review a pattern of a later peak in NSE concentrations in inflicted TBI as compared to non-inflicted TBI. Sandler et al. [12] and Kövesdi et al. [14] note that NSE also has limitations and its use may be limited by a false positive in the setting of hemolysis and extracranial sources.

\section{Myelin basic protein (MBP)}

As with S100B and NSE, Berger et al. [11] found that peak MBP correlated with outcome more strongly than initial concentrations and the correlation is stronger with earlier (0-3 months after injury) outcomes. Initial MBP concentrations correlated with outcome only in the younger children.

In a Sandler et al. [12] review, serum levels of MBP are noted to have good specificity but poor sensitivity for TBI.
Prior study cited in this review reported that in inflicted and non-inflicted TBI specificity and sensitivity of initial peak MBP concentrations is 96 and $44 \%$, respectively. Higher initial and later peak concentration was seen in children with inflicted TBI.

\section{Glial fibrillary acid protein (GFAP)}

Žurek and Fedora's study [10] is the first study measuring GFAP in children with TBI. Comparable to findings with NSE and S100B, GFAP levels at admission showed statistically significant correlations with neurologic follow-up at 6 months post-injury. Findings from a previous study showing that GFAP is not increased in multiple trauma patients without TBI are also noted, indicating this biomarker could be brain-specific. In their review, Kövesdi et al. [14] make mention of prior studies where higher concentrations of GFAP has been found in adults with TBI and an unfavorable outcome.

\section{Imaging markers}

Computed tomography remains an essential part of the acute trauma work-up in TBI because of its ability to rapidly detect lesions requiring surgical intervention. Advanced MRI techniques including diffusion weighted imaging (DWI), diffusion tensor imaging (DTI), susceptibility weighted imaging (SWI), and MR spectroscopy (MRS) increase the ability to detect TBI and potentially improve outcome prediction.

In their 2008 article Suskauer and Huisman [15•] state that the global burden of diffuse axonal injury (DAI) was demonstrated with greater sensitivity using fluid attenuated inversion recovery (FLAIR) sequences that may be more important than localized injury regarding outcome prediction. The DWI is also helpful in detecting DAI in children and, as noted by Galloway et al. [16], the contrast between uninjured immature brain and injured tissue is improved greatly with the use of this technique. In their group of 37 children with TBI, DWI revealed a greater extent and degree of abnormality than T2-weighted or FLAIR sequences, and those with poor outcome had lower mean apparent diffusion coefficient (ADC) values compared to those with good outcomes. Average total brain ADC values alone were best at predicting good versus poor clinical outcome and did so correctly in almost $84 \%$ of cases at 6-12 months post-injury.

Oni et al. [17••] demonstrated that frontal lobe DTI indices of fractional anisotropy (FA), ADC, and radial diffusivity are sensitive to injury severity measured by GCS with the strongest correlation being with radial diffusivity. Significant correlation was found between DTI measures in the frontal lobe and GOS scores at 3 months 
post-injury (Fig. 1). Higher FA and lower radial diffusivity correlate with good outcome. Suskauer and Huisman [15•] noted that other investigators have found that increasing FA over time after TBI may be indicative of functional recovery.

Multiple authors $[18,19]$ have compared imaging modalities and assessed their relative sensitivity and specificity in detecting injury and in outcome prediction. Sigmund et al. [19] found T2, FLAIR, and SWI sequences provided more accurate assessment of injury severity and outcome prediction. The CT scans did not show a significant difference in lesion count or volume of lesions in normal, mild impairment or poor outcome groups and showed a much lower lesion volume than MRI or SWI. All MRI sequences (T2, FLAIR, and SWI) showed significant differences in lesion extent when outcome groups were compared and SWI revealed a higher number of lesions than other MR sequences. Beauchamp et al. [18] also found SWI was more sensitive in detecting lesions than other MRI techniques or CT. A subset of patients with negative CT did not get imaged acutely because of low clinical suspicion and were later found to have parenchymal lesions with MRI and/or SWI highlighting the problem with CT in outcome prediction. Lesion number and volume identified by SWI appear to correlate with global and neurocognitive outcomes after pediatric TBI [15•].

In Suskauer and Huisman's [15•] review of evidence regarding MR spectroscopy, H-MRS data shortly after TBI

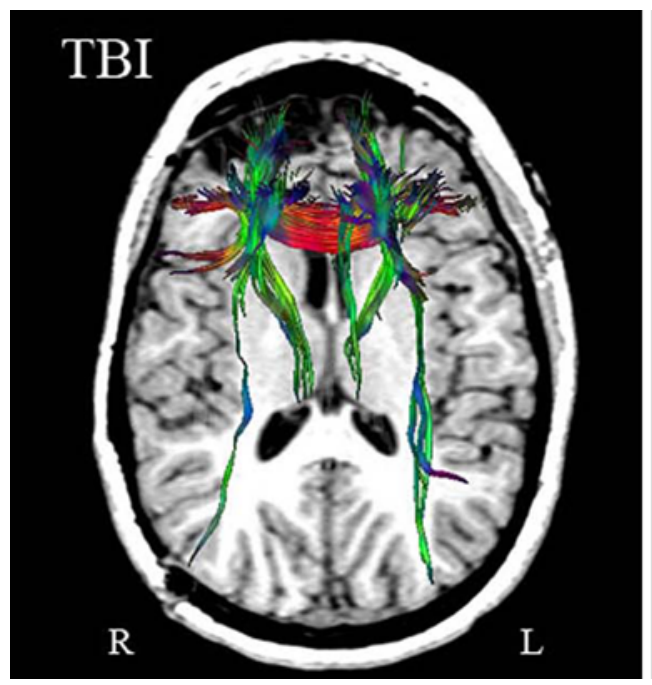

Fig. 1 Diffusion tensor imaging tractography overlaid on a T1-weighted axial image (left) from a 12-year-old girl who had sustained a severe traumatic brain injury after falling from the back of a truck moving at a low speed (initial Glasgow Coma Scale score 1/4 7), striking the back of her head, and sustaining frontal contusions. Tractography and T1-weighted MRI from an age- and sex-matched child with orthopedic injury at approximately the same level are on the right. There is extensive frontal encephalomalacia in the child with traumatic brain injury at 3 months post injury, but the real significance of the frontal damage is seen when tractography maps are has been shown to have a predictive value for long-term behavioral and cognitive outcomes. Several studies have shown decreases in $N$-acetyl aspartate after TBI and neurometabolite abnormalities have been predictive of overall outcome in pediatric TBI.

\section{Rehabilitation approaches}

Gordon and de Maggio [20] reviewed rehabilitation approaches for children with acquired brain injury. They note the wide variability of pediatric rehabilitation practices, family and child-centered care, and reintegration into the community, including school. They review a number of interventions including constraint induced movement therapy, bimanual training, strength training, treadmill training, virtual reality, transcranial magnetic stimulation, motor imagery, mirror therapy, cognitive rehabilitation, pharmacotherapy, botulinum toxin A, orthotics, serial casting and concluded that additional investigation is needed for all of these interventions relative to acquired brain injury.

\section{Technology applications to rehabilitation}

Advances in technology provide an opportunity to support rehabilitation of children and adolescents with traumatic brain injury in new ways. The use of virtual reality,

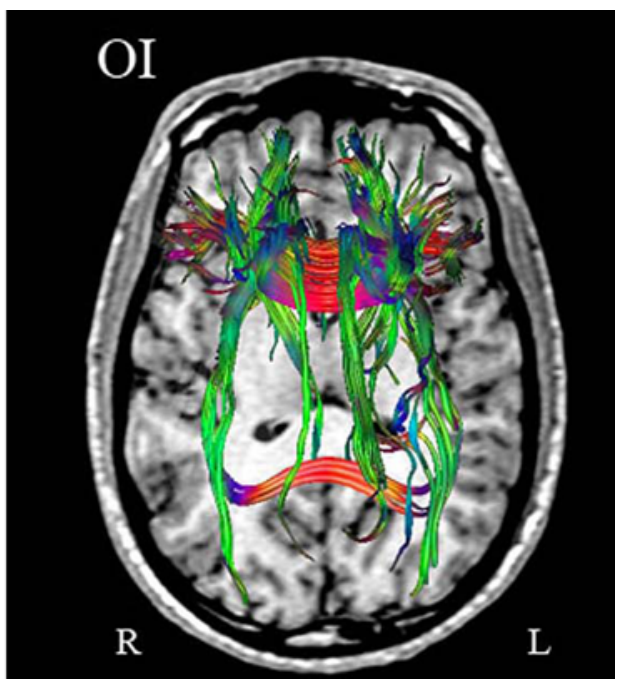

created by seeding identical areas in the coronal plane, at a level just anterior to the genu of the corpus callosum in both children. The normal tracts from the frontal region at this level course posteriorly via central white matter pathways as well as the cingulum. Extensive interhemispheric projection across the anterior corpus callosum that includes an extensive network of integrated bifrontal pathways can be visualized in the child with orthopedic injury. All of these pathways are reduced and thinned in the child with traumatic brain injury. Note at this level, in the child with traumatic brain injury, there was also an absence of callosal fibers across the posterior corpus callosum [17••] 
computer-assisted technology, and personal digital assistants in the form of cognitive orthoses for rehabilitation are all being explored. To date, studies tend to be small and to be lacking in strength of evidence, but trends appear to be emerging.

\section{Virtual reality}

Virtual reality (VR) can provide an artificial environment for people with acquired brain injury to practice functional activities and to model or copy functional patterns of movement to improve performance of activities of everyday life and other neurocognitive tasks. Advantages of VR include the possibility of therapist control of the parameters of the rehabilitation activity, increased access and more opportunities for repetition of the tasks, and immediate feedback.

Feedback from VR can be delivered through a variety of neurologic pathways with visual, auditory, and haptic feedback. One report suggests that modulating the intensity of the feedback, providing variability and indirect competition can optimize participation [21].

Researchers have also proposed that VR can serve to enhance cognitive rehabilitation by providing an enriched environment as described in animal models. It is suggested that children often experience undesirable periods of inactivity from both cognitive and physical activity perspectives during the course of acute rehabilitation. More physical activity, social interaction, and an enriched environment could be provided by a VR option that might benefit neurocognitive rehabilitation [22•].

A pediatric rehabilitation therapist's use of gaming system such as the $\mathrm{Wii}^{\mathrm{TM}}$ in acquired brain injury populations and reasons for utilizing a gaming system has been described. The rationale for this VR use has included enhancing motivation and ongoing participation in the therapy activity. However, there are limitations such as the inability to modulate the system to minimize overstimulation or modify the difficulty level to match the patient's abilities post acquired brain injury [23] Another recent publication describes the $\mathrm{Wii}^{\mathrm{TM}}$ in balance therapy. The authors found that this tool motivated children and it could be coupled with both verbal feedback and physical guidance from the therapists [24]. Use of the PlayStation 3 and 5DT sensing gloves for home-based rehabilitation of the hand in children, including one with TBI, has also been reported. The authors reported that this adolescent demonstrated increased endurance and functional hand use [25].

\section{Smart technology}

Another trend makes use of personal smart technology in the form of devices like pagers, phones, and tablets.
DePompei et al. [26] reported on a long-term follow up study of a small number of school-aged individuals with a history of TBI or intellectual disability who made use of a personal digital assistant or the smartphone technology. Although the subjects required assistance for programming the devices, they preferred reminders from the device rather than a person throughout their days, and continued to use the devices more than a year after initiation of use. Others have also reported increased independence observed for these individuals. They conclude the article with recommendations for clinical use of this type of tool.

\section{Computer assisted technology}

Online applications for support of families with children who have experienced TBI have been reported. Antonini et al. [27] describe the results with an online positive parenting skills program for parents of children with TBI. Skype was used for videoconferencing and additional online sessions were available. Participants reported that the sessions were helpful and easy to access.

\section{Long-term outcomes}

Anderson et al. [28•] recently published a prospective longitudinal study of a group of 40 children with TBI at ages 2-7 that they compared to a group of 16 healthy controls and at 12 and 30 month follow up as well as at 10 years post injury. MRI images were obtained for the TBI subjects at 10-year follow-up. As anticipated, those with severe TBI had the poorest outcomes and the greatest deficits in the cognitive domain, particularly in adaptive, executive and social abilities. A younger age was linked to lower white matter volume at 10 years, but it was unrelated to functional outcomes. As noted in previous studies, family function predicted social and behavioral outcomes and pre-injury adaptive function was predictive of 10-year adaptive abilities. The authors concluded that this data supported that recovery is poorer with injury to the very young and that the immature brain does not result in an increased likelihood of recovery.

In a discussion of plasticity after early brain insult, Anderson et al. [29] conclude that neither plasticity nor vulnerability theories explain the range of functional outcomes seen and that multiple factors including the extent and severity of injury, age, and environmental influences such as family, sociodemographic factors and interventions influence outcome as well. Evaluation at too young an age will prevent the identification of problems such as executive dysfunction as those skills do not emerge until later in life, supporting the need for long-term follow-up in children with TBI.

Another report about attention problems after TBI included the 40 children noted above. Those with severe 
TBI had continuing concerns with attention, both those that develop early and later developing skills. Again, a comparison group of 19 non-injured controls was included. Factors that contributed to attention at 10 years after injury included age at injury and acute IQ [30].

Crowe et al. [31] reported on the outcomes of accidental TBI in 53 children injured prior to age 3 and assessed an average of 40 months after injury. They were compared to a group of 27 children without injury. Children with moderate and severe TBI (grouped together) had significantly lower IQ scores than the control group (although still within the average range) but no differences were seen on parent behavior ratings. No group differences were seen for processing speed as measured by coding on the Wechsler Preschool and Primary School Intelligence Scale-Third Edition coding subtest. Consistent with previous research, predictors of long term intellectual, behavioral and social function after early TBI were socioeconomic status, family function, and parental mental health.

Consistent use of reliable outcome measures is one of the challenges to understanding the long-term sequellae of TBI in children. Paget et al. [32] reported on the use of the King's Outcome Scale for Childhood Head Injury in a retrospective study of clinic reports of 97 children followed by a TBI service. Inter-rater reliability was reported to be good (weighted Kappa 0.710) for investigators of various levels of experience. This scale is an adaptation of the Glasgow Outcome Scale and defines five levels of recovery from death to good recovery with pediatric appropriate definitions of the various levels. The KOSHI score did show some change over time. Twenty-three percent of children under age 8 had a decrease in the score over time (all were under age 3.5 at the time of injury) and reasons for the decrease in the score included intellectual disability not identified during early follow up, new behavioral problems, and the development of late onset seizures.

Rivera et al. [33•] reported on disability seen at 3, 12, and 24 months after TBI in children and adolescents in a large prospective cohort study that included children younger than 18 years at the time of TBI and a comparison group with an arm injury. Outcome measures included health-related quality of life, adaptive skills, and participation. Cause of injury varied with severity of injury (fallsMild 56.6, moderate 34.1, and severe $23.1 \%$, with being a motor vehicle occupant as the most common cause in the moderate and severe TBI groups.) At 3, 12 and 24 month follow up children in the moderate and severe TBI groups had PedsQL, Adaptive Behavior Assessment SystemSecond Edition and Child and Adolescent Scale of Participation scores that were significantly lower than baseline and at 24 months lower than the comparison group as well.
Ganesalingam et al. [34] reported executive function and social competence in young children 6 months after TBI. Children were aged 3 years 0 months-6 year 11 months when they sustained a moderate or severe TBI. A control group included children hospitalized with orthopedic injury. Executive function was rated using the Behavior Rating Inventory of Executive Function (BRIEF) and the Child Behavior Questionnaire. Social competence was assessed using Adaptive Behavior Assessment System (ABAS) and the Preschool and Kindergarten Behavior Scales- Second Edition. They concluded that poorer performance on neuropsychological tests of complex executive functions after severe TBI might be related to impairments that indicate poor self-regulation, metacognition, and effortful control. Higher levels of behavioral aspects of executive functions were related to higher levels of social competence and these results suggest that reduced social competence following TBI may be related to deficits in behavioral expression of executive functions. Also, those with severe TBI demonstrated poorer parent ratings of social competence, suggesting that they are less able to communicate and behave appropriately in social interactions. Individuals with moderate TBI did not perform differently than the control group for social competence.

The risk of epilepsy after TBI in children and young adults based on a population study was reported by Christensen et al [35]. Data was collected from the Danish National Hospital Register and Danish Civil Registration System. Analysis was dichotomized to mild and severe brain injury. Mild brain injury was based on the American Congress of Rehabilitation Medicine criteria. The relative risk of epilepsy was two times higher after mild brain injury and seven times higher after severe brain injury compared to no brain injury for all ages. Brain injury was associated with an increased risk of epilepsy in all age groups but was highest among those older than 15 at injury. They also found that those with a brain injury and a family history of epilepsy had a higher risk than those without a family history of epilepsy.

Headache is a commonly reported symptom after traumatic brain injury. In a study of children ages 5-17, Blume et al. [36] found that the prevalence of headache at 3 months after injury was significantly higher in those with TBI compared to controls with an arm injury.

Sullivan and Riccio [37] published a review of language functioning and deficits following pediatric TBI in 2010. They concluded that language deficits experienced by children post-TBI are quite variable and that comparison of outcomes is complicated by the lack of standard assessments or categorization of TBI. They also noted the lack of information about the interventions used to address language deficits. 


\section{Conclusions}

Pediatric TBI is a commonly occurring disorder, but a need for increased evidence regarding outcomes continues. The NIH working groups have been developing standardized naming, definitions, and data structure for clinical research variables including as it relates to pediatric TBI resulting in several publications defining core and supplemental elements for several domains [38, 39]. Use of these elements could facilitate increased evidence in pediatric TBI.

Disclosure LE Krach: Consultancy and grants/grants pending with Medtronic, Inc.; N Aldahondo: none; A Sinner: none; and S Quigley: none.

\section{References}

Papers of particular interest, published recently, have been highlighted as:

- Of importance

•- Of major importance

1. CDC. Injury Prevention and Control: Traumatic Brain Injury. http://www.cdc.gov/traumaticbraininjury/statistics.html\#A. Accessed 3 Dec 2012.

2. McKinlay A, Grace RC, Horwood LJ, Fergusson DM, Ridder EM, MacFarlane MR. Prevalence of traumatic brain injury among children, adolescents and young adults: prospective evidence from a birth cohort. Brain injury. 2008;22(2):175-81. http://www.ncbi.nlm.nih.gov/pubmed/18240046.

3. Rivara FP, Ennis SK, Mangione-Smith R, MacKenzie EJ, Jaffe $\mathrm{KM}$. Quality of care indicators for the rehabilitation of children with traumatic brain injury. Arch phys med rehabil. 2012; 93(3):381-5.e9. http://www.ncbi.nlm.nih.gov/pubmed/22280892.

4. Zumsteg JM, Ennis SK, Jaffe KM Mangione-Smith R, MacKenzie EJ, Rivara FP. Quality of care indicators for the structure and organization of inpatient rehabilitation care of children with traumatic brain injury. Arch phys med rehabil. 2012;93(3): 386-93.e1. http://www.ncbi.nlm.nih.gov/pubmed/22280893.

5. - Rivara FP, Ennis SK, Mangione-Smith R, MacKenzie EJ, Jaffe KM.Variation in adherence to new quality-of-care indicators for the acute rehabilitation of children with traumatic brain injury. Arch phys med rehabil. 2012;93(8):1371-6. http://www.ncbi. nlm.nih.gov/pubmed/22446294. This reference includes the quality of care indicators for acute rehabilitation of children with TBI that were developed and documents differences in how many of the indicators are met among different types of providers.

6. Pappa L, Robinson G, Oli M, Pineda J, Demery J, Brophy G, et al. Use of biomarkers for diagnosis and management of traumatic brain injury patients. Expert Opin Med Diagn. 2008;2(8): 937-45.

7. Bouvier D, Fournier M, Dauphin J-B, Amat F, Ughetto S, Labbé $A$, et al. Serum S100B determination in the management of pediatric mild traumatic brain injury. Clin chem. 2012;58(7): 1116-22. http://www.ncbi.nlm.nih.gov/pubmed/22529109.

8. Geyer C, Ulrich A, Gräfe G, Stach B, Till H. Diagnostic value of S100B and neuron-specific enolase in mild pediatric traumatic brain injury. J neurosurg Pediatr. 2009;4(4):339-44. http://www. ncbi.nlm.nih.gov/pubmed/19795965.
9. Bechtel K, Frasure S, Marshall C, Dziura J, Simpson C. Relationship of serum S100B levels and intracranial injury in children with closed head trauma. Pediatrics. 2009;124(4):e697-704. http://www.ncbi.nlm.nih.gov/pubmed/19786430.

10. Žurek J, Fedora M. The usefulness of S100B, NSE, GFAP, NF-H, secretagogin and $\mathrm{Hsp} 70$ as a predictive biomarker of outcome in children with traumatic brain injury. Acta neurochir. 2012; 154(1):93-103; discussion 103. http://www.ncbi.nlm.nih.gov/ pubmed/21976236.

11. Berger RP, Beers SR, Richichi R, Wiesman D, Adelson PD. Serum biomarker concentrations and outcome after pediatric traumatic brain injury. J neurotrauma. 2007;24(12):1793-801. http://www.ncbi.nlm.nih.gov/pubmed/18159990.

12. Sandler SJI, Figaji AA, Adelson PD. Clinical applications of biomarkers in pediatric traumatic brain injury. Child's nerv syst. 2010;26(2):205-13. http://www.ncbi.nlm.nih.gov/pubmed/19902 222.

13. Chiaretti A, Barone G, Riccardi R, Antonelli A, Pezzotti P, Genovese O, et al. NGF, DCX, and NSE upregulation correlates with severity and outcome of head trauma in children. Neurology. 2009;72:609-16.

14. Kövesdi E, Lückl J, Bukovics P, Farkas O, Pál J, Czeiter E, et al. Update on protein biomarkers in traumatic brain injury with emphasis on clinical use in adults and pediatrics. Acta neurochir. 2010;152(1):1-17. http://www.ncbi.nlm.nih.gov/pubmed/196529 04.

15. - Suskauer SJ, Huisman T a GM. Neuroimaging in pediatric traumatic brain injury: current and future predictors of functional outcome. Dev disabil res rev. 2009;15(2):117-23. http://www. pubmedcentral.nih.gov/articlerender.fcgi? artid=3167090\&tool $=$ pmcentrez\&rendertype $=$ abstract. This article is a comprehensive review of different imaging techniques used in TBI and how they relate to functional outcome.

16. Galloway NR, Tong KA, Ashwal S, Oyoyo U, Obenaus A. Diffusion-weighted imaging improves outcome prediction in pediatric traumatic brain injury. J neurotrauma. 2008;25(10):1153-62. http://www.ncbi.nlm.nih.gov/pubmed/18842104.

17. •- Oni MB, Wilde EA, Bigler ED, McCauley SR, Wu TC, Yallampalli R, et al. Diffusion tensor imaging analysis of frontal lobes in pediatric traumatic brain injury. J child neurol. 2010; 25(8):976-84. http://www.pubmedcentral.nih.gov/articlerender. fcgi artid=3227397\&tool=pmcentrez\&rendertype=abstract. Excellent example of imaging differences with TBI vs. control.

18. Beauchamp MH, Ditchfield M, Babl FE, Kean M, Catroppa C, Yeates KO, et al. Detecting traumatic brain lesions in children: CT versus MRI versus susceptibility weighted imaging (SWI). J neurotrauma. 2011;28(6):915-27. http://www.ncbi.nlm.nih.gov/ pubmed/21501069.

19. Sigmund GA, Tong KA, Nickerson JP, Wall CJ, Oyoyo U, Ashwal S. Multimodality comparison of neuroimaging in pediatric traumatic brain injury. Pediatr neurol. 2007;36(4):217-26. http://www.ncbi.nlm.nih.gov/pubmed/17437903.

20. Gordon AL, Di Maggio A. Rehabilitation for children after acquired brain injury: current and emerging approaches. Pediatr neurol. 2012;46(6):339-44. http://www.ncbi.nlm.nih.gov/pubmed/226336 27.

21. Wille D, Eng K, Holper L, Chevrier E, Hauser Y, Kiper D, et al. Virtual reality-based paediatric interactive therapy system (PITS) for improvement of arm and hand function in children with motor impairment-a pilot study. Dev neurorehabil. 2009;12(1):44-52. http://www.ncbi.nlm.nih.gov/pubmed/19283533.

22. • Penn PR, Rose FD, Johnson D a. Virtual enriched environments in paediatric neuropsychological rehabilitation following traumatic brain injury: Feasibility, benefits and challenges. Dev neurorehabil. 2009;12(1):32-43. http://www.ncbi.nlm.nih.gov/ 
pubmed/19283532. This article provides an overview of VR and potential uses in pediatric rehabilitation.

23. Levac D, Miller P, Missiuna C. Usual and virtual reality video game-based physiotherapy for children and youth with acquired brain injuries. Phys occup ther pediatr. 2012;32(2):180-95. http://www.ncbi.nlm.nih.gov/pubmed/21942894.

24. Tatla SK, Radomski A, Cheung J, Maron M, Jarus T. Wiihabilitation as balance therapy for children with acquired brain injury. Dev neurorehabil. 2012;1-15. http://www.ncbi.nlm.nih. gov/pubmed/23231377.

25. Burdea GC, Jain A, Rabin B, Pellosie R, Golomb M. Long-term hand tele-rehabilitation on the PlayStation 3: benefits and challenges. Conf Proc IEEE Eng Med Biol Soc. 2011;2011:1835-8. http://www.ncbi.nlm.nih.gov/pubmed/22254686.

26. Depompei R, Gillette Y, Goetz E, Xenopoulos-Oddsson A, Bryen D, Dowds M. Practical applications for use of PDAs and smartphones with children and adolescents who have traumatic brain injury. NeuroRehabilitation. 2008;23(6):487-99. http://www. ncbi.nlm.nih.gov/pubmed/19127002.

27. Antonini TN, Raj SP, Oberjohn KS, Wade SL. An online positive parenting skills programme for paediatric traumatic brain injury: feasibility and parental satisfaction. Journal of telemedicine and telecare. 2012;18(6):333-8. http://www.ncbi.nlm.nih.gov/pub $\mathrm{med} / 22941332$.

28. - Anderson V, Catroppa C, Godfrey C, Rosenfeld J V. Intellectual ability 10 years after traumatic brain injury in infancy and childhood: what predicts outcome? J neurotrauma. 2012;29(1): 143-53. http://www.ncbi.nlm.nih.gov/pubmed/22022835. This article provides objective information about the outcomes of children 10 years after traumatic brain injury and correlates with those outcomes.

29. Anderson V, Spencer-Smith M, Wood A. Do children really recover better? Neurobehavioural plasticity after early brain insult. Brain. 2011;134(Pt 8):2197-221. http://www.ncbi.nlm. nih.gov/pubmed/21784775.

30. Catroppa C, Anderson V, Godfrey C, Rosenfeld J V. Attentional skills 10 years post-paediatric traumatic brain injury (TBI). Brain injury. 2011;25(9):858-69. http://www.ncbi.nlm.nih.gov/pub $\mathrm{med} / 21714623$.

31. Crowe LM, Catroppa C, Babl FE, Anderson V. Intellectual, behavioral, and social outcomes of accidental traumatic brain injury in early childhood. Pediatrics. 2012;129(2):e262-8. http://www.ncbi.nlm.nih.gov/pubmed/22271692.

32. Paget SP, Beath AWJ, Barnes EH, Waugh M-C. Use of the King's Outcome Scale for Childhood Head Injury in the evaluation of outcome in childhood traumatic brain injury. Dev neurorehabil. 2012;15(3):171-7. http://www.ncbi.nlm.nih.gov/pub med/22582847.

33. • Rivara FP, Koepsell TD, Wang J, Temkin N, Dorsch A, Vavilala MS, et al Disability 3, 12, and 24 months after traumatic brain injury among children and adolescents. Pediatrics. 2011;128(5): e1129-38. http://www.ncbi.nlm.nih.gov/pubmed/22025592. This article provides information about outcomes at various time points post TBI.

34. Ganesalingam K, Yeates KO, Taylor HG, Walz NC, Stancin T, Wade S. Executive functions and social competence in young children 6 months following traumatic brain injury. Neuropsychology. 2011;25(4):466-76. http://www.pubmedcentral.nih.gov/ articlerender.fcgi artid $=3125456 \&$ tool=pmcentrez\&rendertype $=$ abstract.

35. Christensen J, Pedersen MG, Pedersen CB, Sidenius P, Olsen J, Vestergaard M. Long-term risk of epilepsy after traumatic brain injury in children and young adults: a population-based cohort study. Lancet. 2009;373(9669):1105-10 doi:10.1016/S0140-6736 (09)60214-2.

36. Blume HK, Vavilala MS, Jaffe KM, Koepsell TD, Wang J, Temkin N, et al. Headache after pediatric traumatic brain injury: a cohort study. Pediatrics. 2012;129(1):e31-9. http://www.ncbi. nlm.nih.gov/pubmed/22144708.

37. Sullivan JR, Riccio C A. Language functioning and deficits following pediatric traumatic brain injury. Appl neuropsychol. 2010; 17(2):93-8. http://www.ncbi.nlm.nih.gov/pubmed/20467948.

38. Adelson PD, Pineda J, Bell MJ, Abend NS, Berger RP, Giza CC, et al. Common data elements for pediatric traumatic brain injury: recommendations from the working group on demographics and clinical assessment. J neurotrauma. 2012;29(4):639-53. http:// www.ncbi.nlm.nih.gov/pubmed/21939389.

39. Miller A C, Odenkirchen J, Duhaime A-C, Hicks R. Common data elements for research on traumatic brain injury: pediatric considerations. J neurotrauma. 2012;29(4):634-8. http://www.nc bi.nlm.nih.gov/pubmed/22091862. 